\title{
Rasgos constitucionales de la protección del embrión. Una introducción*
}

\author{
Emanuele Rossi \\ Catedrático de Derecho Constitucional \\ Scuola Superiore Sant'Anna
}

Recibido: 02.05.12

Aceptado: 31.05 .12

Resumen: En este artículo se hace una comparación del concepto de embrión a nivel europeo, haciendo especial énfasis a la doctrina y jurisprudencia italiana. Se hace referencia a las consecuencias y problemas que se pueden derivar de un determinado trato a embriones y por último se hace una disertación sobre la conveniencia de un status unitario, aplicable en todas las circunstancias e independientemente de la "utilización" que se quiera hacer del embrión o de las circunstancias en las que sea considerado por las disposiciones normativas

Palabras clave: Embrión, jurisprudencia, estatuto, Tribunal de Justicia, países europeos.

Abstract: In this paper, there is a comparison between the concept of embryo at European level, with particular emphasis on Italian doctrine and jurisprudence. Also, there is an analysis of the consequences and problems that can arise from a particular treatment embryo and finally becomes a dissertation on the desirability of a unitary status, applicable in all circumstances and regardless of the "use" or the embryo or the circumstances under consideration by the regulatory provisions tries.

Key words: Embryo, jurisprudence, statute, Court of Justice, European coun-

Sumario: 1 . El concepto de embrión en la disciplina nacional y europea, y las perspectivas abiertas por la sentencia del Tribunal de Justicia Oliver Brüstle vs. Greenpeace.-2. Las diferencias entre la regulación de los diferentes Estados europeos y el dilema de la definición "natural" o "convencional".- - 3. El estatuto jurídico del concebido/embrión: las posibles alternativas propuestas por la doctrina jurídica y la jurisprudencia italiana.-4. ¿Es posible distinguir entre "categorías" de embriones? Los problemas relacionados con el distinto trato (con consecuencias paradójicas) de los embriones in vitro respecto a los embriones in vivo.5. La condición particular de los llamados embriones supernumerarios y

* Ponencia presentada al VI Seminario italo español de Derecho Constitucional, "La construcción jurisprudencial de la Europa de los valores: jueces, criterios ético-políticos y razonamiento jurídico entorno al inicio de la vida", Madrid 29-30 de marzo de 2012. Traducción de Josep Ma Castellà (UB) y Carlos Vidal (UNED). 
las posibles soluciones jurídicas a su condición. - 6 . A modo de conclusión: ¿un estatuto o más estatutos del embrión según los ámbitos a que se refiera?

\section{EL CONCEPTO DE EMBRIÓN EN LA DISCIPLINA NACIONALY EUROPEA, Y LAS PERSPECTIVAS ABIERTAS POR LA SENTEN- CIA DEL TRIBUNAL DE JUSTICIA OLIVER BRÜSTLE VS. GRE- ENPEACE}

Preguntarse por los perfiles constitucionales de la protección del embrión requiere, en primer lugar, definir la noción de la entidad cuyos perfiles de garantía se quiere indagar, o comprender de qué se habla cuando se alude al embrión. Sustancialmente, ¿a qué (o a quién) nos referimos cuando usamos el término "embrión”? ¿Se corresponde esta expresión íntegramente con la de "concebido"?

Para responder a esta pregunta, tratando de superar el estado de desorientación que asume el jurista frente a ella ${ }^{1}$, debemos hacernos la pregunta sobre el contexto en el que queremos responder y, en particular, para nosotros los juristas, del ordenamiento jurídico de referencia: por ejemplo podríamos responder sobre la base del ordenamiento comunitario, o el del Consejo de Europa, o asimismo el de cada Estado en particular. Pero incluso antes podríamos preguntarnos si existe una noción "natural" -o "científica" y por tanto "objetiva" - capaz en consecuencia de condicionar las opciones de los distintos sistemas jurídicos. Esta última pregunta requeriría preguntarse, de forma preliminar, por la fuerza del "derecho natural" y su relación con el derecho escrito. Böckenförde habló al respecto de una "falacia naturalista", entendida como la operación que deriva del dato de hecho constatable por las ciencias naturales (es decir, del ser), postulados y cualidad normativa (es decir, el deber ser) ${ }^{2}$. Claramente, un esfuerzo que va mucho más allá de los límites de este trabajo.

Por lo tanto he decidido escoger el camino del derecho positivo, y responder a la pregunta partiendo de las disposiciones normativas, constatando a continuación que en el estado de cosas "no es posible hablar de un estatuto jurídico unificado y universal del embrión, sino que debe reconocerse que cada ordenamiento concibe el "propio" embrión en cuanto lo somete a sus propias leyes" ". La reciente sentencia Oliver Brüstle vs Greenpeace (de la

${ }^{1}$ Considera que en esta materia el papel del jurista corre el riesgo de "entrar en confusión" A. CELOTTO, "Procreazione assistita e tutela della persona. Profili di costituzionalità", en A. BARENGHI (ed.), Procreazione assistita e tutela della persona, Cuadernos de la Rivista di diritto civile, Padua, 2011, 109.

${ }^{2}$ E. W. BOCKENFORDE, Dignità umana e bioetica, Brescia, 2010, 46.

${ }^{3}$ A. SCHUSTER, "Lo status giuridico dell'embrione in ottica comparata", en C. CASONATO, C. PICIOCCHI, P. VERONESI, Percorsi a confronto. Inizio vita, fine vita e altri problemi, Padua, 2009, 259. 
cual se tratará a continuación) lleva a pensar que, contrariamente a lo sucedido en el Consejo de Europa, la perspectiva de la Unión Europea es importante para lograr un nivel común mínimo para los países que forman parte de ella.

¿Qué se entiende, por lo tanto, con el término "embrión” en el contexto de la Unión Europea?

La respuesta se puede deducir de las previsiones contenidas en la Directiva 98/44/CE del Parlamento Europeo y del Consejo, de 6 de julio de 1998, sobre la protección jurídica de las invenciones biotecnológicas. El artículo 6.2 , establece que se consideran no patentables, en particular:

a) los procedimientos de clonación de seres humanos;

b) los procedimientos de modificación de la identidad genética germinal del ser humano;

c) la utilización de embriones humanos con fines industriales o comerciales.

Esta última previsión, por lo tanto, plantea el problema de definir qué se entiende por "embriones humanos", por lo menos a los efectos de dicha Directiva. Bajo este perfil se promovió una cuestión ante el Tribunal de Justicia por Oliver Brüstle contra Greenpeace, en la que el demandante era el titular de una patente alemana en relación con células progenitoras neurales: dicha patente fue anulada por el Tribunal federal de patentes alemán, a solicitud de Greenpeace, en cuanto células obtenidas a partir de células madre embrionarias humanas y de procedimientos para la producción de tales células progenitoras. Contra esta decisión, el interesado apeló ante el Bundesgerichtshof, el cual elevó una cuestión prejudicial al Tribunal de Justicia, considerando - entre otras cosas- que "la interpretación del concepto de embrión humano no puede ser sino europea y unitaria". Esta consideración ha permitido por lo tanto al Tribunal Europeo abordar el tema ex profeso ${ }^{4}$.

Antes de examinar las respuestas del Tribunal, merecen ser mencionadas las conclusiones del Abogado General Yves Bot, en las que se ha puesto la cuestión sobre qué deba entenderse por "embrión" con claridad y precisión, especificándose al respecto la cuestión de si en el ámbito de dicho concepto se debe considerar incluidas:

a) todas las etapas de desarrollo de la vida humana desde la fecundación del óvulo, o si por el contrario deben cumplirse otras condiciones posteriores, como, por ejemplo, el alcance de una determinada etapa de desarrollo;

b) los óvulos humanos no fecundados en que se ha trasplantado un núcleo proviniente de una célula humana madura y los óvulos humanos no fecundados, estimulados por la partenogénesis para separarse y desarrollarse;

c) las células madre derivadas de embriones humanos en la etapa del blastocisto.

${ }^{4}$ Y resolverlo con la citada sentencia de 18 de octubre 2011 C-34/10 Oliver Brüstle vs. Greenpeace. 
Preparatoria de esa pregunta es esta otra, esto es, si el concepto de embrión humano tiene, o debe tener una acepción comunitaria, como ha sostenido el Bundesgerichthof, o si en cambio la definición del concepto entra en el margen de discrecionalidad de los Estados.

En la motivación del pronunciamiento, el Tribunal de Justicia responde primero a la pregunta sobre la necesidad de definir un concepto comunitario, y lo hace motivando sobre la formulación de la Directiva, la cual, si bien por un lado no proporciona ninguna definición de embrión humano, tampoco se remite a los derechos nacionales, en lo concerniente al significado a atribuir a estos términos. Y, por lo tanto, es necesario designar "un concepto autónomo del Derecho de la Unión, que debe ser interpretado de manera uniforme en el territorio de esta última".

Volviendo, pues, al fondo de la cuestión, el Tribunal llega a una definición amplia de embrión: con tal término se debe entender, en opinión del Tribunal, "cualquier óvulo humano desde la fecundación, a cualquier óvulo humano no fecundado en el cual haya sido implantado el núcleo de una célula humana madura y cualquier óvulo humano no fecundado que, a través de la partenogénesis, ha sido inducido a dividirse y a desarrollarse", mientras considera competente al juez nacional para "determinar, en vista de la evolución de la ciencia, si una célula madre derivada de un embrión humano en el estado de blatocisto" constituye un embrión o no.

Es cierto que la misma sentencia especifica que dicha interpretación vale "al único propósito de determinar el alcance de la prohibición de patentabilidad" establecido por la normativa europea, y que el Tribunal se preocupa de dejar claro que no quiere abordar "temas de naturaleza médica o ética", sino que se limita a "una interpretación jurídica de las disposiciones de la Directiva": y sin embargo, es evidente que este concepto no puede dejar de tener impacto tanto sobre el desarrollo del ordenamiento comunitario, como sobre las opciones de los Estados y los operadores económicos. También hay que recordar que ese proncunciamiento se sitúa en claro contraste con la jurisprudencia del TEDH, que por su parte había subrayado la existencia sobre este punto de un margen de apreciación de la autonomía legislativa respectiva de los Estados, bajo la premisa de la falta de consenso sobre la definición científica y legal del embrión humano ${ }^{5}$ : así, permanece abierta la cuestión sobre cuál sea el nivel institucional al que deba reconocerse la responsabilidad de definir lo que es el embrión, a la espera de respuestas a la sentencia del Tribunal de Justicia por parte de los jueces nacionales.

${ }^{5}$ CEDU, Evans v. UK, n. 6339/05, 7 de marzo de 2006, que confirma sustancialmente Vo vs. France, n. 53924/00, 8 de julio de 2004, y en la que el Tribunal de Estrasburgo excluye la aplicabilidad de la tutela ofrecida al art. 2 CEDU a la vida prenatal. Sobre tal pronunciamiento v. V. SILVESTRI, "Il quesito sul diritto alla vita dell'embrione e /o del feto ex art. 2 CEDU rimane ancora insoluto nella sentenza Evans c. Regno Unito della Corte Europea dei Diritti dell'Uomo", in www.rivistaaic.it, 25 de julio de 2006. 


\section{LAS DIFERENCIAS ENTRE LA REGULACIÓN DE LOS DIFEREN- TES ESTADOS EUROPEOS Y EL DILEMA DE LA DEFINICIÓN "NATURAL" O "CONVENCIONAL"}

Si del nivel europeo o internacional se pasa al de los países individuales, encontramos diferencias de perspectiva nada desdeñables.

Como ha señalado el Abogado General Yves Bot en la memoria citada, en la legislación de los Estados miembros "se distinguen dos grandes grupos, el primero que considera que el embrión humano existe desde la fecundación (por ejemplo Estonia, Alemania, Reino Unido), y el segundo que considera que esto sucede en el momento en que el óvulo fecundado se implanta en el endometrio o mucosa uterina (España y Suecia)".

Debe señalarse al respecto que la adopción de un concepto amplio (o incluso el más amplio que sea posible) de embrión no significa garantizar una forma de protección adecuada: de hecho a veces puede ser al contrario. Así, por ejemplo, si el Reino Unido es el Estado que adopta una definición de embrión más omnicomprensiva, al mismo tiempo está entre los "que menos protegen el embrión", ya que permite su uso para fines de investigación científica e incluso parece abrir espacios para la introducción de quimeras.

Para los países en los que no se afirma la coincidencia entre la noción de embrión y la de concebido se prevé la condición del denominado pre-embrión: así en España, por ejemplo, donde la ley establece que por tal debe entenderse "el embrión constituido in vitro formado por el grupo de células resultantes de la división progresiva del ovocito desde que es fecundado hasta 14 días más tarde" "fase del desarrollo embrionario que abarca desde el momento en que el ovocito fecundado se encuentra en el útero de una mujer hasta que se produce el inicio de la organogénesis, y que finaliza a los 56 días a partir del momento de la fecundación, exceptuando del cómputo aquellos días en los que el desarrollo se hubiera podido detener". Se trata de una línea reconstructiva que constituye aplicación y desarrollo del famoso Informe Wornock, el cual ha establecido al cumplirse el día 14 el reconocimiento del embrión y por tanto de su derecho a la vida (se ha hablado al respecto de teorías transaccionales) ${ }^{9}$.

${ }^{6}$ A. SCHUSTER, Lo status giuridico, cit., 264, el cual precisa che tal planteamiento de menor tutela se traduce en la tendencia a poner el embrión en la ponderación con otros intereses.

${ }^{7}$ En el sentido del art. 1.2 de la Ley 14/2006, sobre técnicas de reproducción humana asistida; art. 3 s) de la Ley 14/2007, de Investigación Biomédica.

${ }^{8}$ Por el art. 3 l) de la Ley 14/2007, de Investigación Biomédica.

${ }^{9}$ Siguiendo tal línea reconstructiva, la misma fase pre-embrional podría ser ulteriormente concretada (por ejemplo desde el acto de la fecundación hasta el estadio de 8 células; de tal momento a la formación del troboflasto; del troboflasto al estadio de preparación para la implantación del óvulo y de este momento al estadio de la post implantación (del $6^{\circ}$ al $14^{\circ}$ día). Cfr. al respecto A. SPADARO, "Cellule staminali e fecondazione assistita: i dubbi di un giurista", en Rass. dir. pubbl. eur., n. 1/2005, 71 ss. 
Otros países, sin embargo, identifican el embrión con el "concebido", identificando la concepción con el momento de la fecundación (aunque los nuevos hallazgos científicos llevan a considerar la fecundación como un proceso dinámico que tiene lugar en el tiempo, y no como un evento simple e instantáne $\mathrm{o}^{10}$ ). La reciente Constitución húngara parece, como se sabe, incluso haber constitucionalizado este principio, afirmando que "Todo hombre tiene derecho a la vida y a la dignidad humana, la vida del feto debe ser protegida desde la concepción". En realidad, la fórmula utilizada puede dejar abiertas algunas dudas de interpretación: distinguiedo entre "el hombre" que tiene el derecho a la vida y a la dignidad humana y el "feto" al que, evidentemente, no puede reconocerse los mismos derechos (de lo contrario no tendría sentido la diferenciación), pero cuya vida está protegida. En cualquier caso, la intención del constituyente húngaro es clara al imponer una garantía del embrión "desde la concepción", y por lo tanto excluir toda posibilidad de gradación en la protección entre una fase y la otra de la vida prenatal (mientras que no está clara en mi opinión -como se ha dicho- la diferencia entre la tutela pre y post-natal, por lo que se acaba de decir).

En este contexto general, y con el propósito de establecer una definición jurídica, el problema es elegir el punto de referencia: si este debe ser "un elemento naturalista bien definido, detallado y por tanto incontrovertible como ha sido durante mucho tiempo el nacimiento y lo que es hoy, por ejemplo, el momento de la unión de los gametos, o bien este puede ser de tipo jurídicoconvencional, correspondiendo a la norma jurídica, sin otros puntos de apoyo naturalistas, determinar el momento a partir del cual se considera o se presume que está en presencia de una persona" ${ }^{11}$. Pregunta que sigue a una reflexión de fondo, llevada a cabo por Violini y Osti: “'hay un momento en el tiempo antes del nacimiento en el que surge el titular de un derecho a la vida y a la salud, pero dicho momento no es determinable por naturaleza sino que caso por caso puede la norma sustituir a la naturaleza identificando la misma el momento del cambio?"'12.

Todo esto plantea algunas preguntas, en cierto modo preliminares al problema de la definición jurídica: ¿se remite ésta a la voluntad de los Estados? ¿Corresponde al derecho, y por lo tanto, en primer lugar al legislador determinar lo que es "correcto" y lo que es "equivocado" en esta materia? ${ }^{13} \mathrm{Y}$ en el caso de

${ }^{10}$ L. PALAZZANI, Il concetto di persona tra bioetica e diritto, Turín, 1996, 43.

${ }^{11}$ L. VIOLINI, A. OSTI, "Le linee di demarcazione della vita umana", en M. CARTABIA (ed.), I diritti in azione, Bolonia, 2007, 189.

${ }^{12}$ L. VIOLINI, A. OSTI, "Le linee di demarcazione...", cit., 205.

${ }^{13}$ En sentido claramente afirmativo F. RIMOLI, "Bioetica. Diritti del nascituro. Diritti delle generazioni future", in R. NANIA, P. RIDOLA, I diritti costituzionali, I, Turín, 2001,348, para el cual "la determinación del momento en que el ser vivo se convierte en sujeto de derecho, adquiriendo la capacidad jurídica y por tanto la idoneidad para ser centro de imputación de derechos, constituye el fruto de una opción del ordenamiento". Para un cuadro general de las dificultades que ponen al derecho, y en particular a la definición 
respuesta afirmativa a la pregunta anterior, surge el problema de cuál debe ser el nivel en el que definir este concepto: ¿es la legislación europea, tal como es interpretada por sus jueces, o la nacional, también ésta en la interpretación de los sujetos legitimados para ello? Y por último ¿hay principios constitucionales nacionales, o incluso principios constitucionales europeos?

Así pues la pregunta “¿Qué se entiende por embrión?” remite a otra: ¿sobre qué base se puede responder a la misma? Como se dirá en la conclusión, la sentencia Brüstle del Tribunal de Justicia podría tener efectos muy significativos en la respuesta que se de a estas preguntas.

\section{EL ESTATUTO JURÍDICO DEL CONCEBIDO/EMBRIÓN: LAS PO- SIBLES ALTERNATIVAS PROPUESTAS POR LA DOCTRINA JURÍ- DICA Y LA JURISPRUDENCIA ITALIANA}

La segunda pregunta que debemos hacernos es: ¿cuál es el estatuto jurídico de esta entidad que no logramos definir de forma unitaria?

Una vez más, para responder a la pregunta se debe definir el ordenamiento jurídico de referencia, tal vez incluso en este caso tampoco vamos a obtener una respuesta definida y unívoca, pero sin duda hay que tener en cuenta la diversidad de planos, y en todo caso que en los ordenamientos europeos no existe un estatuto común. Así como debemos entender si la respuesta debe ser proporcionada de conformidad con la legislación vigente, emblemática es en este sentido la posición, relativa al ordenamiento italiano, de un jurista notorio, que conecta el estatuto a lo que establece la norma, y en particular a la del Código Civil: "para el concebido se ha hablado de un "derecho a la vida", pero la expresión, si del discurso de los moralistas y los teólogos quiere pasar al de los juristas, choca contra la norma que la hace coincidir con el nacimiento de la "adquisición de la capacidad jurídica y por tanto de los derechos (art. $1 \mathrm{CC})$ " ${ }^{14}$. Si se sigue este camino, entonces, la respuesta debe darse en relación con lo que prevén las leyes de cada ordenamiento (a excepción de la posibilidad de reducirlos a una orientación unitaria y coherente, es decir, independientemente del ámbito de regulación en el que tiene lugar la protección del embrión).

En general, sin embargo, podemos decir que la respuesta a esa pregunta, creo que en cada uno de los planos indicados, puede oscilar entre tres posiciones teóricamente identificables.

Para la primera de ellas, el concebido se considera "persona" a todos los efectos ("uno de nosotros", dice Busnelli en sentido figurado ${ }^{15}$ ). Elementos

de "persona", algunas aplicaciones del saber científico en el campo humano v. A. SANTOSUOSSO, "Persone fisiche e confini biologici: chi determina chi", en A. D'ALOIA (ed.), Diritti e Costituzione. Profili evolutivi e dimensioni inedite, Milán, 2003, 141 ss.

${ }^{14}$ P. RESCIGNO, "Personalità (diritti della)", en Enciclopedia del diritto, vol. XXX, Milán, 1983, 7.

${ }^{15}$ F. D. BUSNELLI, "Il problema della soggettività del concepito a cinque anni dalla legge sulla procreazione medicalmente assistita”, en Nuova Giur. civ. commentata, 2010, II, 185. 
que apoyan esta posición se pueden encontrar en algunos textos normativos, también de alcance internacional, como por ejemplo la Declaración sobre los Derechos del Niño aprobada por la Asamblea General de la ONU en 1959 en Nueva York, en cuyo preámbulo está escrito que "el niño, debido a su falta de madurez física e intelectual, necesita de una protección y de cuidados especiales, incluida una protección legal apropiada, tanto antes como después del nacimiento". Una definición que no define el dies a quo para la condición de niño (ni por tanto el concepto de embrión), pero que sin duda requiere retrotraer la noción a una fase anterior al nacimiento.

Probablemente la definición más clara de la posición en cuestión está en la Convención Americana de Derechos Humanos (22 de noviembre de 1969), conocida como Pacto de San José, que establece que "cada ser humano es persona" (art. 1.2 ) y que "toda persona tiene derecho al respeto de su vida" que debe ser garantizado "en general desde el momento de la concepción" (art. 4): de donde la respuesta parecería clara, ya sea con relación al punto en cuestión, como también al anterior (es decir, en lo relativo a la definición de embrión). Con respecto a la legislación italiana, se llega a una conclusión similar sobre la base del artículo 1 de la Ley núm. 40/2004, que se basa en el supuesto de "la equivalencia perfecta entre la persona humana y embrión"16.

Incluso en ausencia de una normativa específica, se ha sostenido de forma autorizada que la posición indicada puede e incluso debe mantenerse en virtud de la protección fijada en el plano constitucional a la dignidad humana: así Böckenförde, en relación con lo que establece la Constitución alemana, considera que "si el respeto a la propia dignidad debe valer para cada hombre en cuanto tal, la misma debe serle atribuida desde el principio, desde el primer momento de su vida, y de aquí debe extenderse no sólo después de un lapso de tiempo, a quien él -no protegido contra la instrumentalización y tratamiento ad libitum - tal vez por casualidad ha sobrevivido", y este "primer inicio de la vida humana propia del hombre en sí mismo formado y desarrollado se halla en la fecundación, no más tarde" ${ }^{17}$.

${ }^{16}$ C. TRIPODINA, "Statuto dell'embrione vs. libertà di ricerca scientifica: un conflitto risolto in modo incostituzionale", en www.nuvole.it, 1 . El art. 1 de la ley n. 40/2004 establece, recuérdese, que dicha ley "asegura los derechos de todos los sujetos involucrados, incluido el concebido". Antes de la entrada en vigor de tal normativa -y por tanto sobre la base de elementos no de derecho positivo sino con base en la fuerza de principios obtenidos de la ciencia o de la interpretación y traducción jurídica de la misma- un documento del Comité nacional de bioética de 1996 ("Identità e statuto dell'embrione umano") llegaba a conclusiones análogas: en el mismo se afirmaba, en efecto, "el deber moral de tratar el embrión humano, desde la fecundación, como una persona". Afirmación que podría también ser leída como contraria a la identificación embrión/persona (si debe ser tratado como persona, significa que no es persona), sino que no obstante creo quisiera significar una equiparación efectiva.

17 E. W. BOCKENFORDE, Dignità umana e bioetica, cit., 55. 
La identificación embrión=persona comporta, por lo menos a nivel general, el reconocimiento al embrión de todos los derechos inviolables de la persona (si bien con las especificaciones necesarias de conformidad con el canon de la razonabilidad), y en primer lugar el derecho a la vida, tal y como claramente lo ha expresado la Corte Constitucional italiana: "Así también se ha fortalecido la idea, inherente a la Constitución italiana, en particular en el art. 2, según la cual el derecho a la vida, entendido en su acepción más lata, debe ser incluido entre los derechos inviolables, esto es, entre los derechos que ocupan en el ordenamiento una posición, por así decirlo, privilegiada, en tanto que pertenecen -por utilizar la expresión de la sentencia núm. 1146 de 1988- "a la esencia de los valores supremos sobre los que se funda la Constitución italiana".

Esta posición ha sido criticada en relación con diferentes puntos de vista.

Dejando de lado las afirmaciones apodícticas y totalmente inaceptables de cierta bioética de Estados Unidos, que agrupa como "no-personas humanas" a todas las formas de vida humana no reconducibles a un modelo productivista $^{18}$, se han avanzado posiciones que tienden a diferenciar la condición del embrión de la de persona ("uno de nosotros"). Entre ellos podemos recordar la tesis que niega la calificación de persona humana al embrión sobre la base de la suposición de que el embrión carece de toda capacidad de obrar, criterio decisivo que "no permite calificarlo aún como persona", entendida ésta como un ser social y relacional, en oposición a las concepciones individualistas-atomistas ${ }^{19}$. Otras posiciones hacen referencia más directa -en cuanto a la legislación italiana- a lo que establece el art. 1 del Código Civil, que atribuye la titularidad de la capacidad jurídica en el momento del nacimiento, y que subordina la protección de los derechos reconocidos por la ley a favor del concebido a tal acontecimiento. En apoyo de esta línea de interpretación hay un pasaje de la motivación de una sentencia no reciente de la Corte Constitucional italiana, que en 1975 declaró que "... no hay equivalencia entre el derecho no solo a la vida, sino también a la salud de quien ya es persona, como la madre, y la protección del embrión que todavía no se ha convertido en persona" (sentencia núm. 27/1975).

${ }^{18}$ Según H. T. ENGELHARDT Jr., Manuale di bioetica (ed. it. a cargo de M. Meroni), Milán, 1999, 126, son ejemplos de no-personas "los niños, los deficientes mentales graves y aquellos que están en coma sin esperanza".

${ }^{19}$ A. SPADARO, Cellule staminali, cit. También para A. PATRONI-GRIFFI, "L'embrione umano: dimensione costituzionale, modelli legislativi e bilanciamenti ragionevoli", en www.forumcostituzionale.it., 9 de junio de 2005, no es posible atribuir la calificación de persona a un ser vivo "en un momento en que no tiene ninguna conciencia de si mismo, ningún sistema nervioso, ninguna célula nerviosa, ninguna posibilidad de sentir emociones, vida de pensamiento y ningún tipo de capacidad relacional”. 
Una segunda concepción considera sin embargo al concebido como una parte de cuerpo de la madre y, en consecuencia, esencialmente en la categoría de una res, en particular como "pars viscerum matris"20.

A pesar de que esta posición me parece que hoy en día no se sostiene con tal claridad, sin embargo subyace a las posiciones que abordan el tema desde el punto de vista del derecho de los padres: "Los padres que deciden procrear imponen, ipso facto, la vida (...) a un sujeto que no existe, y no puede, obviamente, expresar su consentimiento a una decisión que le concierne inmediata y completamente. Ante tal forma -inevitable- de prevaricación, la idea de cualquier derecho del sujeto futuro revela toda su inconsistencia". Así que debido a que "la responsabilidad de la elección se sitúa plenamente en la esfera de los padres, la eventual decisión de interrumpir un embarazo o de recurrir a técnicas avanzadas de procreación (...) no puede de ninguna manera ser considerada una ilicitud, ni más prevaricatoria sobre la voluntad (hipotética y no valorable) del nascituro de lo que lo haya sido la propia decisión de procrear" 21 .

Estas consideraciones están en la base de las legislaciones sobre el aborto que definen los límites de la licitud de la interrupción voluntaria del embarazo mediante el sistema de "plazos", o bien, que propugnan su liberalización en un plazo determinado desde la concepción, sin necesidad de de aducir causas de justificación, tal vez constitucionalmente merecedores de protección. Esta solución, ciertamente "dejando a la mujer cualquier decisión sobre la oportunidad de recurrir al aborto durante el plazo permitido, termina negando que el feto sea portador de valores jurídicamente protegidos frente a la madre ${ }^{22}$ : por lo tanto, lo considera una res.

Esta posición, sin embargo, ha sido considerada contraria -al menos en términos de principio- con los principios constitucionales de la jurisprudencia constitucional de varios países europeos: en primer lugar de los tribunales constitucionales alemán e italiano ${ }^{23}$, y luego el portugués y el español ${ }^{24}$, tam-

${ }^{20}$ Sobre tal aspecto, y para referencias a la doctrina italiana sobre el punto, cfr. R. ROMBOLI, "sub art. 5 ”, in A. PIZZORUSSO, R. ROMBOLI, U. BRECCIA, A DE VITA, Persone fisiche, Bolonia-Roma, 1988, 290 ss.

${ }^{21}$ F. RIMOLI, "Bioetica", cit., 347. En sentido opuesto, entre otros, C. E. TRAVERSO, Interruzione volontaria della gravidanza, Milán, 1978, 41-42, nota 15, para el cual “es posible configurar un derecho a autodeterminarse libremente en orden a la procreación, (...) sin embargo no es posible invocar el derecho a disponer del propio cuerpo y del propio embarazo".

${ }^{22}$ Así T. PADOVANI, "Procreazione (dir. pen.)”, en Enc. diritto, vol. XXXVI, Milán, $1987,976$.

${ }^{23}$ De la Corte Constitucional italiana véase la sentencia núm. 27/1975, ya citada; del Tribunal Constitucional alemán ver la casi coetánea sentencia de 22 de febrero de 1975.

${ }^{24}$ Sentencia del Tribunal Constitucional de Portugal de 19 de marzo de 1984, en Foro it., 1985, IV, 294; sentencia del Tribunal Constitucional español 53/1985, de 11 de abril, en Foro it., 1985, IV, 280. 
bién han reconocido la existencia de una entidad "tercera" respecto a la madre (aunque colocada en el cuerpo de ésta), como tal titular de derechos propios y no asimilable a una res.

Coherentemente, la legislación italiana reconoce en algunas previsiones la existencia de derechos de naturaleza personal al producto de la concepción ${ }^{25}$ : por ejemplo el art. $254 \mathrm{CC}$, en que está prevista la posibilidad de reconocer como hijo natural propio a un concebido, posibilidad que no se concilia bien con la inexistencia jurídica de este último; así el DPR 3.11.2000, n. 396 -en materia de estado civil-, el cual establece que el reconocimiento de un hijo nasciturus puede hacerlo el padre junto con la mujer embarazada o con posterioridad al de ésta última con su consentimiento (de acuerdo con lo dispuesto en el artículo 250.3). A esto se añade que, según la jurisprudencia, es posible proceder a la custodia del hijo solamente concebido a la madre en caso de separación, sobre la base de la necesidad de proteger los intereses del nascituro $^{26}$.

En vista de la respuesta insatisfactoria ofrecida al problema de la naturaleza del embrión por las dos primeras soluciones, se ha formulado una tercera, que me parece que es la más seguida, al menos en el ámbito jurídico: "el concebido (sea embrión o feto), si bien no se ha convertido todavía en persona, sin embargo ya es un ser humano, por lo tanto, un verdadero sujeto" 27. Por lo tanto, no puede ser considerado "uno de nosotros", pero tampoco puede ser considerado como una "cosa" (aunque fuese "cosa viviente").

Pero, ¿cómo se puede definir esta "entidad" jurídicamente, pasando del elemento negativo (lo que no es: ni persona ni cosa) a una reconstrucción en términos positivos?

Manteniéndonos en el plano jurídico, en primer lugar hay que decir que a esta entidad se le reconocen (a nivel constitucional, y en virtud de la jurisprudencia constitucional de varios países) algunos derechos, por otro lado reconocidos y confirmados también en el nivel de la legislación ordinaria. Como por ejemplo declaró la Corte Constitucional italiana, "la protección del concebido -que ya es relevante para el derecho civil (artículos 320, 339, 687 $\mathrm{CC}$ - tiene fundamento constitucional. El artículo 31.2 de la Constitución impone expresamente la "protección de la maternidad" y, más en general, el art. 2 de la Constitución reconoce y garantiza los derechos humanos inviolables, entre los que no puede omitirse, incluso con sus características particulares propias, el estatuto jurídico del concebido".

La afirmación anterior permite precisar algunos aspectos, dignos de ser profundizados: en primer lugar, que la "tutela de la maternidad" no se orienta solo a la atención de la madre o, más en general, de la maternidad (incluso

\footnotetext{
${ }^{25}$ Así, entre otros, E. PALMERINI, "Il concepito e il danno non patrimoniale", en Nuova giur. civ. comm., 2011, I, 1272 ss.

${ }^{26}$ Trib. Catania 9 de diciembre de 1991, en Dir. fam.,1992,251 ss.

${ }^{27}$ Así textualmente A. SPADARO, Cellule staminali, cit.
} 
"potencial"), sino también al producto de la concepción (que, como tal, debería ser considerado también como un producto de la paternidad). En segundo lugar, que el embrión/concebido es titular de derechos inviolables que corresponden a todo "hombre", si bien "con sus propias características". Eso significa, pues, que la tutela del embrión no se hace equivalente a la de la persona: como afirmó la Corte Constitucional "la tutela del embrión no es sin embargo absoluta, sino limitada por la necesidad de encontrar una justa ponderación con la tutela de las exigencias de la procreación" (sentencia n. 151/2009). Análoga me parece la perspectiva española, para la cual el concebido no es "persona", sino "bien jurídico constitucionalmente protegido".

Volviendo a la cuestión, es evidente que hay una cierta convergencia en considerar que el embrión pueda ser considerado como un "sujeto". Retomando la doctrina autorizada que había ensayado la distinción entre sujeto y persona $^{28}$, una importante sentencia de la Corte de Casación italiana ha sostenido que "el concebido es sujeto, no persona" con el fin de reconocer al individuo-concebido la titularidad de un interés protegido, por otro lado no posible $\sin$ la atribución de la subjetividad ${ }^{29}$. Esta operación requiere, por otra parte, un cambio conceptual nada fácil, o la superación de la ecuación sujeto=persona: de hecho, sobre la base de la sentencia, "debe hoy entenderse por subjetividad jurídica un concepto sin duda más amplio del de capacidad jurídica de las personas físicas..., con la consiguiente no absoluta coincidencia desde un punto de vista jurídico, entre sujeto y persona" ${ }^{30}$. Y por lo tanto, si "toda persona es sujeto, no todo sujeto es persona".

Lo anterior aporta ciertamente un punto de claridad, pero todavía relativa: ¿Qué es pues el "sujeto"? y ¿qué tipo de "sujeto" es el concebido? La misma sentencia de la Corte de Casación afirma que, de hecho, la subjetividad que corresponde al concebido de acuerdo con la ley es una subjetividad "que se diga específica, especial, atenuada, provisional o parcial".

En términos más específicos se señala que este sujeto se caracteriza por no tener ninguna capacidad de obrar (que sería propia de la persona); mientras que en relación con la capacidad jurídica, hay diferentes posiciones. Quien cree que el concebido tiene capacidad jurídica (y esto también sobre la base de lo establecido por el artículo 1 de la Ley n. 40/2004, en virtud del cual -se recuerda- la misma ley "garantiza los derechos de todas los sujetos involucrados, incluyendo al concebido"), aunque "menor"31, o

${ }^{28}$ G. OPPO, “Ancora su persona umana e diritto”, en Riv. dir. civ., 2007, I, 259 ss.

${ }^{29}$ Cass. n. 10741/2009, comentada por G. BALLARANI, "La Cassazione riconosce la soggettività giuridica del concepito: indagine sui precedenti dottrinali per una lettura “integrata" dell'art. 1 c.c.", en Dir. famiglia e persona, 2009, fasc. 3, pt. I, 1180 ss.

${ }^{30}$ G. BALLARANI, "La Cassazione riconosce...", cit., 1186.

${ }^{31}$ Elemento que comportaría un redimensionamiento de la misma noción de capacidad jurídica: como sostiene F. D. BUSNELLI, "L'inizio della vita umana", en Riv. dir. civ., 2004, I, 563: “quien ve en la intervención del legislador la atribución de una subjetividad 
"en sentido parcial o iuspublicístico" ${ }^{32}$; otros, en cambio, se plantean la hipótesis de una "subjetividad por prefiguración de la persona", posición elaborada para contraponerse tanto a la tesis de la extensión de la capacidad jurídica a la vida prenatal, como a la tesis de la subjetividad del concebido como mera afirmación desprovista de concretos efectos jurídicos ${ }^{33}$. Esta tesis tendría como precedente relevante el artículo 12 del Código Civil francés, en la redacción dada en las leyes sobre la bioética, que reconoce la "primauté de la personne" y asegura "le respect de tout etre humain dès le commencement de la vie", donde la diferencia estaría entre "reconocer" y "respetar".

Siguiendo esta línea, se podría por tanto argumentar que el concebido tiene una subjetividad, pero específica y propia (y distinta de la de "persona" en el sentido propio de la palabra): él gozaría pues de ciertos derechos reconocidos también por la legislación y la jurisprudencia (y que pueden ser ampliados y restringidos por las mismas), tales como el derecho a la vida, a la identidad genética, a la salud-establecido por la jurisprudencia en materia de tutela resarcitoria- e incluso a las relaciones familiares (como lo considera la jurisprudencia sobre indemnización por daños).

Se trata de una conclusión que pretende graduar la titularidad de los derechos desde la óptica de la no uniformidad de su catálogo: por analogía -aunque mutatis mutandis- con la jurisprudencia, también la constitucional, que incluso dentro de la misma "categoría" de "persona" distingue respecto a la pertenencia de los derechos garantizados por la Constitución en relación con el quantum de la protección que les presta. Piénsese en la distinción entre ciudadanos y no ciudadanos, con base en la cual, de acuerdo con la jurisprudencia constitucional, a los segundos deben reconocerse los derechos inviolables, pero solo con respecto a su núcleo fundamental, mientras que no se impide que el legislador distinga, incluso dentro de la protección de un derecho inviolable, entre el quantum de protección ofrecida a todos y cuánta se puede reconocer solo a los ciudadanos ${ }^{34}$. En esta perspectiva, así como en la relación entre embrión/sujeto y persona, el criterio rector es el de la razonabilidad, puesto que, como ha afirmado la Corte Constitucional, en esta materia el legislador ordinario tiene "una amplia

a individuos (que continúan estando) privados de capacidad jurídica según el Código Civil está obligado a considerar que el dogma de la ecuación subjetividad/capacidad jurídica se ha roto definitivamente, evidenciando la inidoneidad de la noción del Código de capacidad jurídica de agotar la búsqueda de individuos merecederores de protección en relación con los principios fundamentales de la Constitución".

${ }^{32}$ Así A. SPADARO, Cellule staminali, cit.

${ }^{33}$ F. D. BUSNELLI, "Ai confini della soggettività", en F. TESCIONE (ed.), Persona e soggetto. Il soggetto come fattispecie della persona, Nápoles, 2010, 47.

${ }^{34}$ Para referencias a la jurisprudencia constitucional italiana sobre este punto permítaseme remitirme a E. ROSSI, "Commento all'art. 2", en Commentario alla Costituzione, R. BIFULCO, A. CELOTTO y M. OLIVETTI (eds.), vol. I, Turín, 2006, pp. 47 ss. 
discrecionalidad, limitada, en el marco de la adecuación a la Constitución, solo por el vínculo de que sus opciones no sean manifiestamente irrazonables" (sentencia núm. 62/1994). Por lo tanto, la identificación de las situaicones jurídicas que se han de reconocer al embrión solo podrá ser el resultado de opciones discrecionales fundadas sobre efectivas "diferencias de situaciones fácticas y de las consiguientes valoraciones jurídicas" (Corte Constitucional. núm. 104/1969).

\section{4. ¿ES POSIBLE DISTINGUIR ENTRE “CATEGORÍAS" DE EMBRIO- NES? LOS PROBLEMAS RELACIONADOS CON EL DISTINTO TRATO (CON CONSECUENCIAS PARADÓJICAS) DE LOS EM- BRIONES IN VITRO RESPECTO A LOS EMBRIONES IN VIVO}

Otro aspecto crítico es la posibilidad de atribuir las conclusiones que acabamos de indicar a cada embrión (o concebido, si se prefiere), o si, por el contrario, es necesario diferenciar entre "categorías" de embriones. Me refiero en particular a la distinción entre embriones in vitro (por lo tanto, aún no implantados en el útero materno) y en los embriones in vivo: según algunos, de hecho, solo debería reconocerse la subjetividad a los segundos, y no en cambio a los primeros.

La ley italiana, en verdad, parece dar la vuelta al problema que acabamos de mencionar. La condición de los embriones in vitro es, de hecho, regulada por la ley de fecundación asistida, la cual les garantiza una tutela muy relevante, tanto para impedir la creación de un número de embriones superior al estrictamente necesario (antes de la intervención de la Corte Constitucional con la sentencia núm. 151/2009- dicho número era indicado directamente por la ley, y se establecía la obligación de una implantación única: la Corte consideró estas previsiones irrazonables y potencialmente lesivas del derecho a la salud de la mujer), y así impedir la crioconservación de los embriones producidos (mientras que tras el fallo de la Corte deben conservarse aquellos no implantados por elección médica).

La condición de los embriones in vivo en cambio es disciplinada por la ley de interrupción voluntaria del embarazo, la cual prevé la posibilidad para la mujer de llevar a cabo la interrupción voluntaria del embarazo sustancialmente sin necesidad de motivación en los primeros noventa días ${ }^{35}$. Así, el resultado paradójico de esta situación es que "el embrión es persona en la probeta pero cosa en el útero materno; sagrado e inviolable en el estado de

35 Dentro de dicho plazo, en efecto, si bien la ley subordina la admisibilidad de procedimientos abortivos a determinadas condiciones, "la serie de circunstancias materiales que legitiman la petición de la mujer, además de resultar más bien lábil e incierta, no se acompaña ciertamente por la previsión de alguna forma de verificación vinculante por parte del órgano al que la mujer se ha de dirigir": así T. PADOVANI, Procreazione, cit., 976. 
óvulo fecundado y privado del ámbito materno que le es indispensable para venir al mundo, pero a merced del principio de autodeterminación de la mujer una vez implantado, e incluso una vez que se alcanza el estado fetal", con una situación en general "más que de irrazonabilidad, de esquizofrenia" 36 . A esto se añade una paradoja más: que la eventual implantación del embrión in vitro en el útero materno, y la subsiguiente posible decisión de la mujer de abortar, haría que la "persona" primero se convirtiese en res, y luego fuese suprimida. $\mathrm{O}$, para continuar con las paradojas, que si se desea eliminar un embrión primero hay que implantarlo en el útero, porque solo así se "degrada" su ser como persona.

Así que, por lo que se ha dicho, la situación se invierte con respecto a la perspectiva indicada: en efecto no es a los embriones in vivo a los que se reconoce subjetividad, sino a aquellos in vitro. Eso sería posteriormente contradictorio si se adhiriese a la tesis propuesta que tiende a distinguir las dos situaciones recurriendo a diferencias ontológicas entre los dos "tipos" de embrión: mientras el embrión in vivo estaría en una condición de desarrollo de la vida prenatal, el otro no (aunque haya que considerarlo "un verdadero y propio sujeto posible, un sujeto futuro, un cuasi-suejto" ${ }^{37}$ ). Esta conclusión tiene un fundamento lógico que no es fácil de superar: aun suponiendo que es cierto todo lo que se ha dicho hasta ahora acerca de la subjetividad del embrión y por tanto su catálogo de derechos, es claro que se trata de un sujeto que requiere (en el estado actual del conocimiento científico, por lo menos) de una mujer que lo acoja y lo lleve a buen fin con el parto. Pero igualmente claro es que una conducta de este género no puede ser impuesta ni siquiera a aquella mujer que, junto con su pareja, haya solicitado y obtenido la producción de embriones en la clínica: en efecto ella podría cambiar de opinión más tarde y no querer la implantación. En esta circunstancia es difícil si no imposible -incluso en el plano de los principios constitucionales- imaginar una obligación de actuar sobre el cuerpo de la mujer (al respecto alguno ha hablado incluso de un "estupro medicalizado"38). Estaríamos ante un comportamiento que podría dar lugar a eventuales acciones de responsabilidad contra la mujer y su pareja, las cuales, sin embargo serían inadecuadas para proporcionar ningún tipo de protección al embrión o a los embriones producidos.

${ }^{36}$ Como justamente subraya C. TRIPODINA, Statuto dell'embrione, cit., 6. Análogamente S. CECCANTI, "Procreazione assistita: ci sono tre problemi di costituzionalità", in www.forumcostituzionale.it, 7 de abril de 2004, para quien "la razonabilidad (y primero aun la lógica) lleva consigo un necesario aumento de tutela a medida que se va aproximando al nacimiento y no una protección "a saltos", ignota a cualquier ordenamiento".

${ }^{37}$ F. MODUGNO, "La fecondazione assistita alla luce di principi e della giurisprudenza costituzionale", en Rass. parlamentare, 2005, 371.

${ }^{38}$ D. CARUSI, "In vita, “in vitro", in potenza. Verso una donazione dell'embrione soprannumerario?”, en Riv. crit. dir. priv., 2010, 336. 


\section{LA CONDICIÓN PARTICULAR DE LOS LLAMADOS EMBRIONES SUPERNUMERARIOS Y LAS POSIBLES SOLUCIONES JURÍDI- CAS A SU CONDICIÓN}

Otro aspecto crítico viene dado por la condición de los llamados embriones supernumerarios. Se trata, como se sabe, de los embriones congelados y que no están destinados a implantarse (el número total de ellos, en Italia, es una fuente de incertidumbre, y en las últimas semanas noticias de prensa indican que se habría decidido no recogerlos más en un único centro, como se había decidido anteriormente). Sobre la base de datos proporcionados por el Instituto Superior de la Salud en 2011, en Italia existirían 3.415 embriones crioconservados abandonados por 825 parejas; sin embargo, en el caso de otros 6.079 embriones congelados no es posible localizar a los 1.499 padres. Por tanto, habría aproximadamente 10.000 embriones crioconservados, si bien las cifras son muy aleatorias (de hecho, otros hablan de 20.000 embriones o incluso 27.000).

El problema que estos plantean es un dilema: ¿conservarlos en el estado en que se encuentran, a sabiendas de que están destinados a morir, o utilizarlos con fines de investigación, como se solicita desde el mundo científico (y no solo)? En verdad, habría una tercera posibilidad, que la ley podría prever por lo menos para algunos de ellos ${ }^{39}$ : permitir la "adopción" o la implantación en mujeres que estuvieran disponibles. Hipótesis que de un lado se enfrenta a la prohibición de la fecundación heteróloga establecida por la ley italiana, y que además requeriría una adhesión voluntaria (entre otras cosas, no sólo de una mujer, sino de una pareja, estando la adopción vedada a personas solas). Esta es, por otra parte, la solución adoptada en algunos países europeos, por ejemplo en Francia con la ley núm. 800/2004, donde es posible por parte de las parejas decidir si destinan sus embriones a parejas infértiles, a la investigación o su supresión. Las estadísticas muestran que de los 173.523 embriones congelados de 50.000 parejas, el $50 \%$ han sido abandonados, 37.000 por indicación escrita de sus padres, mientras que en 45.000 casos no ha sido posible localizar a las parejas donantes. Quien ha dejado indicaciones, lo ha hecho así: 10.000 han dado el embrión a parejas infértiles, otros tantos a la investigación, mientras que 17.000 han pedido no conservarlos más (teniendo en cuenta que en Francia, sin embargo, el tiempo límite de crioconservación sin indicación de los padres es de 5 años).

Pero en un sistema jurídico donde la normativa no proporciona condiciones para la utilización de los embriones sobrantes ¿quid juris? Es evidente, por su parte, que una eventual investigación científica sobre dichos embriones

${ }^{39}$ Hay que decir para aquellos que son crioconservados por un número limitado de años, porque para los otros se considera que se ha producido un deterioro tal que los hace inutilizables para el propósito: lo que acentúa la conclusión que se indica en el texto, al menos para estos últimos. 
no se dirigiría a preservar sus vidas, sino eventualmente a producir efectos potencialmente benéficos para ciertas enfermedades y así-siempre potencialmente- para otros sujetos. Las razones que llevan a algunos a creer incorrecto el uso de tales embriones con fines de investigación científica parten de una doble motivación: en primer lugar, que el propósito de la ley no solo es la protección del derecho a la vida de los embriones, sino también de su dignidad, la cual sería violada en el caso de una utilización con fines de investigación científica, y en segundo lugar porque habría de todos modos una diferencia entre dejar morir y la intervención activa con fines eliminatorios. Sobre la base de estas premisas, se ha afirmado que "asumiendo la premisa mayor de que el hombre, sin su consentimiento y con fines que no sean potencialmente positivos incluso para su propia salud, no puede ser objeto de experimentación, y admitiendo la premisa menor de que el embrión es persona humana, la conclusión solo puede ser que incluso los embriones supernumerarios destinados a un fin cierto, pueden ser objeto de investigación experimental" 40 .

Este enfoque me parece, sin embargo, demasiado rígido y viciado de presupuestos ideológicos. En efecto, partiendo de la premisa de que estos embriones están destinados a morir de todos modos y que no hay otra perspectiva para ellos (esta es la premisa lógica del discurso, y no es actualmente controvertida), creo que deberíamos preguntarnos si se protege más su dignitas haciéndolos vegetar en espera de la muerte - cierta e inevitable- o permitir que por medio de ellos otras vidas humanas se pueden salvar y/o mejorar ${ }^{41}$. A mí me parece que la solución, tanto lógica como finalista, de tutela de la vida humana, se orientaría más bien en el sentido de permitir su utilización: regulada, dirigida, acompañada de la necesaria protección, por supuesto, pero no prohibida en nombre de una declaración de principio puramente abstracta. El peligro a que podría dar lugar esa conclusión, o el posible "deslizamiento ético", debe considerarse una preocupación a tener en cuenta, pero no es un obstáculo absoluto: como bien sostiene Spadaro,se trata de una "razón práctica ligada a argumentaciones excesivamente preconcebidas y pesimistas, además de no compatibles (...) con la "idea de la situación", la cual sin embargo debería inspirar cada opción jurídica razonable"42.

${ }^{40}$ C. TRIPODINA, Statuto dell'embrione, cit., 5. Para tal posición v. entre otros, S. BAGNI, "La nuova legge sulla procreazione assistita alla prova della Consulta sarebbe davvero destinata all'aborto?", en www.forumcostituzionale.it

${ }^{41}$ En términos análogos, si bien en mi opinión excesivamente orientados a una utilización de los embriones en cuestión con la finalidad de investigación científica (hasta el punto de considerar inconstitucional la ley italiana sobre fecundación por violación del principio de libertad de invetigación científica) v. G. COLETTA, "Embrioni umani e ricerca scientifica: il caso italiano", en Revista brasileira de Direito Constitucional, en./jul. 2011, 311 ss.

${ }^{42}$ A. SPADARO, Cellule staminali, cit. Según el mismo autor (en La sentenza Brüstle sugli embrioni: molti pregi e... altrettanti difetti), la sentencia del Tribunal de Justicia 
Comparto, por tanto, la solución del art. 18 de la Convención de Oviedo, que prohíbe la creación de embriones in vitro con fines de investigación, admitiendo de manera implícita que la investigación con embriones supernumerarios puede tener lugar cuando ello es permitido por la ley nacional, garantizando en todo caso una protección adecuada del embrión ${ }^{43}$.

\section{A MODO DE CONCLUSIÓN: ¿UN ESTATUTO O MÁS ESTATUTOS DEL EMBRIÓN SEGÚN LOS ÁMBITOS A QUE SE REFIERA?}

Como conclusión parcial a estas consideraciones, quiero formular dos preguntas finales.

La primera de ellas ha atravesado en cierta medida los distintos puntos examinados. $\mathrm{Y}$ es una pregunta que está bien resumida en la sentencia núm. 151/2009 de la Corte Constitucional, donde se lee: "A este respecto, cabe señalar que la jurisprudencia constitucional ha puesto el acento repetidamente en los límites que plantean los logros científicos y experimentales, que están en continua evolución y en los que se funda el arte médico, a la discrecionalidad legislativa". Si el legislador está limitado en su discrecionalidad, no solo por las fuentes constitucionales, sino también (directa o indirectamente) por la ciencia, ¿qué margen tiene para definir un estatuto jurídico de una entidad sobre la que no hay (o tal vez puede cambiar) una evidencia científica? ¿Cuál es, en esencia, el "margen de apreciación" reservado al derecho (y me refiero, en general, a todo el derecho, independientemente de su fuerza normativa: constitucional o legislativa, etc.)?

La segunda pregunta en cierta medida está relacionada con la anterior: ¿es correcta una disciplina jurídica del embrión en sí mismo considerado, y por tanto independientemente del ámbito y para los propósitos para los cuales se ha regulado? En la situación actual se considera, en los distintos países, una disciplina diferenciada en función de que se considere el embrión como destinado a la fecundación asistida o a la experimentación científica, o bien objeto de regulación en el ámbito de la disciplina sobre el aborto. Por lo tanto, no parece que se pueda hablar de un "status" del embrión, sino de tantos estatutos cuantos sean los ámbitos en los cuales se le considere. ¿Es esto correcto?

citada al inicio puede llegar a ser engañosa en relación con dicho aspecto, porque refiriéndose a la producción de células estaminales totipotentes corre el riesgo de impedir la utilización científica de los embriones criocongelados existentes, no adoptables y destinados de todos modos a no ser utilizados de ningún modo, ni tampoco la posibilidad de patentar la producción de tales células estaminales pluripotentes, que no son y no pueden nunca convertirse en embriones: de modo que, según el autor, "parece que el Tribunal de Luxemburgo, más que inspirarse en el "principio de precaución", haya pecado de "exceso de precaución"'".

${ }^{43}$ Sobre tal previsión v., entre otros, G. CATALDI, "La Convenzione del Consiglio d'Europa sui diritti dell'uomo e la biomedicina", en L. CHIEFFI (ed.), Bioetica e diritti dell'uomo, Turín, 2000, 269 ss. 
No estoy seguro de cuál sea la respuesta correcta, o por lo menos la mejor.

Por un lado, exigencias de coherencia lógica y jurídica, así como de garantía del respeto del principio de igualdad y no discriminación, me harían inclinar hacia la necesidad de definir un status unitario, aplicable en todas las circunstancias e independientemente de la "utilización" que se quiera hacer del embrión o de las circunstancias en las que sea considerado por las disposiciones normativas ${ }^{44}$. Por otro lado, es cierto que casi ningún status es uniforme en relación con las circunstancias en las cuales entra en contacto. Dicho en otras palabras, incluso el status es relativo, ya que va ligado a la ponderación que hay que llevar a cabo entre los derechos o los intereses del que el mismo es portador con otros derechos e intereses. Así que incluso si reconstruimos o definimos el estatuto del embrión de manera unitaria, esto no significará que vaya a tener un trato jurídico diversificado en razón del hecho de que esté destinado a la fecundación asistida, o que sea objeto de un embarazo natural y en esta situación pueden sufrir daños u otras eventualiades, $o$ incluso que la mujer que lo lleva pueda decidir si continuar o interrumpir el embarazo.

Esta segunda posición, sin embargo, me parece idónea para justificar la necesidad de definir un status que tenga en cuenta las distintas circunstancias y necesidades de regulación, pero las razones antes mencionadas hacen que me incline hacia la necesidad de una regulación lo más unitaria posible y, sobre todo, que conciba unitariamente el embrión y su estatus jurídico. Por el momento esto puede y debe hacerse en el nivel de los Estados, y sin embargo, la orientación de la jurisprudencia adoptada por el Tribunal de Justicia en el caso Brüstle v. Greenpeace puede ser portadora de consecuencias también sobre este plano: si se consolida la convergencia sobre el concepto de embrión, es posible que en un tiempo más o menos rápido se consolide también la convergencia sobre su disciplina jurídica.

${ }^{44}$ Una problemática análoga surge, en la jurisprudencia constitucional italiana, con referencia a la noción de "muerte", que estaba legislativamente definida de forma diversa según se refiriese a cuerpos destinados al trasplante de órganos o a cuerpos no utilizables a tal fin: la cuestión de constitucionalidad, elevada a la Corte, concluyó con una decisión de devolución al juez a quo porque mientras tanto se había aprobado una nueva ley (ley de 29 de diciembre de 1993, núm. 578) que había uniformado las modalidades de verificación de la muerte (auto núm. 273/1994). 